\title{
Possibilities of Cancer Chemotherapy Based on Transporter-Conscious Drug Design
}

\section{Toshihiko Tashima}

Nippon Pharmaceutical Chemicals Co Ltd, Japan

*Corresponding author: Tashima T, 2-8-18 Chodo, Higashi-Osaka, Osaka 577-0056, Japan, Tel: 8106 6781 0346 ; Fax: 81 06 6787 0771; E-mail: ttashima@nichiri.co.jp

Received date: July 01, 2015; Accepted date: July 25, 2015; Published date: July 27, 2015

Copyright: ( 2015 Tashima T, et al. This is an open-access article distributed under the terms of the Creative Commons Attribution License, which permits unrestricted use, distribution, and reproduction in any medium, provided the original author and source are credited.

\begin{abstract}
Cancer chemotherapy is the treatment method which uses drugs to kill cancer cells or to inhibit their growth. In cancer drug discovery or development, not only anti-cancer drug delivery into target cancer cells across the cell membrane but also acquired anti-cancer drug resistance through the overexpression of ATP-binding cassette (ABC) transporters such as MDR1 (p-glycoprotein) in target cancer cells are serious problems to overcome. However, the use of transport system based on solute carrier (SLC) transporters can solve both problems, because it is wellknown that some types of SLC transporters are overexpressed in cancer cells. Thus, transporter-consciously designed drugs can be transported into cells by such overexpressed SLC transporters, so as not to be excreted by MDR1. Therefore, transporter-conscious drug design is very effective in absorption, distribution, excretion, and toxicity of drugs (ADMET) due to transport systems. In this paper, cancer chemotherapy based on transporterconscious drug design is described.
\end{abstract}

Keywords: Breast Cancer; Cancer chemotherapy; Transporterconscious drug design; Drug design; Drug delivery; Transporter

\section{Abbreviations:}

ABC-transporter: ATP Binding Cassette Transporter; MDR1: Multidrug Resistance 1 (P-glycoprotein); SLC transporter: Solute Carrier Transporter; dtc: Dithiocarbamato; Sar: Sarcosine; Aib- $\alpha$ : Aminoisobutyric Acid; $\mathrm{O}(t$-Bu): tert-butoxy; Val: Valine

\section{Introduction}

Chemotherapy is the treatment for cancer cells by the use of anticancer drugs. However, in drug discovery or development, delivery of anti-cancer drugs across the cell membrane into cancer cells is a crucial problem. Moreover, cancer cells eventually acquire drug resistance through the overexpression of $\mathrm{ABC}$ transporters such as MDR1 (p-glycoprotein). Transporters are the cell membrane proteins which absorb or excrete materials such as nutrients and metabolites and are tissue-specifically expressed. Thus, well-designed compounds based on transporter-conscious design control the absorption, distribution, excretion, and toxicity of drugs (ADMET). Recently, it has been revealed that some types of SLC transporters are overexpressed in cancer cells compared to normal cells. Therefore, the use of their transport systems overcomes the problems such as drug permeability into cancer cells and drug resistance through the overexpression of MDR1 (Figure 1) [1,2]. I have already described intriguing possibilities and beneficial aspects of transporter-conscious drug design [3]. In this review, I will introduce the effective method of cancer chemotherapy based on transporter-conscious drug design.

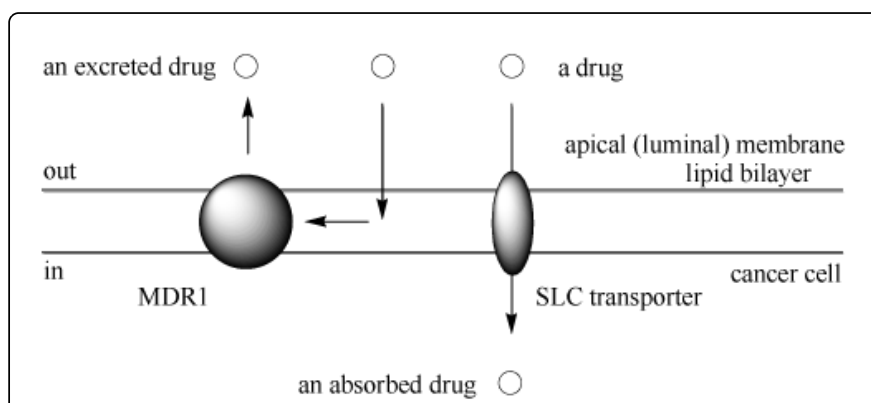

Figure 1: The passage of drugs excreted by MDR1 or absorbed by overexpressed SLC transporter in cancer cells.

\section{Discussion}

\section{Characterization of transporters and their substrates}

Transporter proteins [4], tissue-specifically expressed in the apical or basolateral membranes, play a very important role in regulating physiological homeostasis by transporting materials such as nutrients, metabolites, and waste substances across the cell membrane in the living body. Transporters are divided into two major superfamilies: ABC transporter superfamily and SLC transporter superfamily [5]. In general, $\mathrm{ABC}$ transporters excrete materials such as xenobiotics and SLC transporters absorb materials such as nutrients. MDR1, a representative $\mathrm{ABC}$ transporter, is expressed not only in apical (luminal) membrane of capillary endothelial cells or small intestinal epithelial cells but also largely in drug-resistant cancer cells. MDR1 traps hydrophobic compounds passing through the membrane before they reach the cytoplasmic side and then excretes them to the outside of the cell [6]. Thus, by taking advantage of co-expressed SLC 
transporters, drugs can be delivered into cells across the cell membrane without being excreted by MDR1s (Figure 1). Therefore, transporter-conscious drug design is very important [7].

Transporters are involved in ADMET. Many types of drugs are known to interact with transporter proteins [8,9]. Transporters recognize specifically structural features of their own substrates, respectively. Peptide transporters transport dipeptide and tripeptide, and organic anion transporters (OATs) transport anionic compounds. And then, organic cation transporters (OCTs) transport cationic compounds. On the contrary, the transporter recognition unit of arbitrary transporters can be suggested from the view of the structural commonalities of their known substrates. Transporter-conscious designs are simultaneously substrate-conscious designs.

\section{Specific overexpression of SLC transporters in cancer cells}

It is known that many cancer cells overexpress some types of SLC transporters, compared to normal cells. Thus, such overexpressed SLC transporters can be the target transporters for transporter-consciously designed drug delivery into cancer cells. PEPT1 (peptide transporter 1) is overexpressed in tumor cell lines such as AsPc-1 (pancreas adenocarcinoma), Capan-2 (pancreas adenocarcinoma), HT-1080 (fibrosarcoma), and A549 (lung adenocarcinoma) [10]. Substrates of PEPT1 are dipeptides and tripeptides. Compounds possessing peptide mimic as transporter recognition unit can be transported into cancer cells by PEPT1. GluT1 (glucose transporter 1) is overexpressed in cancer cells, particularly in breast cancer cells compared to healthy breast cells [11,12]. Incorporating glucose mimic as transporter recognition unit into novel compounds may develop cell-permeable anti-cancer drugs due to the glucose transport system. Moreover, although OATP1B1 and OATP1B3, belonging to organic anion transporting polypeptide (OATP), are down-regulated in liver cancer, they are up-regulated in gastrointestinal tract cancer, breast cancer, prostate cancer, and lung cancer [13]. Anionic moieties of designed compounds may function as transporter recognition unit. Accordingly, compounds possessing the substrates' structural commonality of arbitrary transporters specifically overexpressed in target cancer cells as transporter recognition unit may enter into such cancer cells and elicit their activity there.

\section{Implementation of transporter-conscious design}

Transporters recognize the structure of their substrates and subsequently transport them across the cell membrane. Therefore, the structural commonality of arbitrary transporter substrates can shape transporter recognition unit. Actually, transporter-consciously designed compounds were transported into cancer cells based on the transport system. Gold compounds exhibit growth inhibitory activity against cancer cells. [ $\mathrm{Au}^{\mathrm{III}} \mathrm{Br}_{2}(\mathrm{dtc}-\mathrm{Sar}-\mathrm{Aib}-\mathrm{O}(t-\mathrm{Bu}))$ ] (Figure 2) was the conjugate composed of gold (III) complex and peptide mimic as transporter recognition unit and eventually exhibited about 30 -fold more potent growth inhibitory activity than cisplatin in in vitro assay against cisplatin-resistant ovarian adenocarcinoma C13 cell line [14]. This conjugate compound may be transported into cells due to the carrier-mediated transport system based on PEPT1 overexpressed in cancer cells. In addition, oleanolic acid (OA) and its derivatives exhibit anti-cancer activity. Nitric oxide (NO) is involved in apoptosis of cancer cells.L-Val-L-Val-OA-nitrate compound as a prodrug (Figure 2) was the conjugate composed of peptide mimic as transporter recognition unit, OA, and nitrate as NO donor element and exhibited 7 -fold stronger cytotoxic activity than that of cisplatin in in vitro assay against A549 cell line overexpressing PEPT1 [10]. The transportation of this conjugate compound may be based on PEPT1-mediated transport.

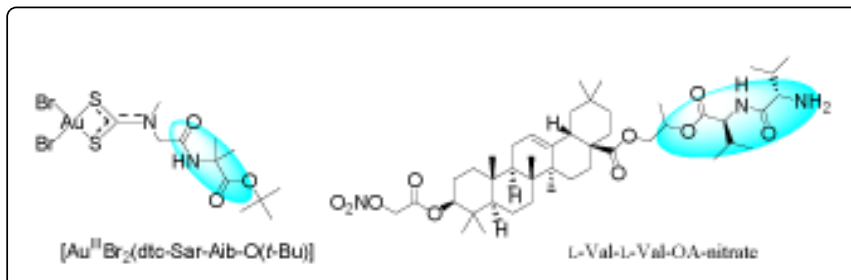

Figure 2: The structures of $\left[\mathrm{Au}^{\mathrm{III}} \mathrm{Br}_{2}(\mathrm{dtc}-\mathrm{Sar}-\mathrm{Aib}-\mathrm{O}(t-\mathrm{Bu}))\right]$ and $\mathrm{L}-$ Val- L-Val-OA-nitrate. Peptide mimics indicated by a blue sphere might act as PEPT1 recognition unit.

By the same strategy based on transporter-conscious drug design, anti-breast cancer drugs may be effectively obtained by using overexpressed SLC transporters such as GluT1, OATP1B1, or OATP1B3 in breast cancer cells. Paclitaxel is an important agent in the treatment of several cancers including breast cancer. Glucoseconjugated paclitaxel derivatives through the ester bond or the ether bond (Figure 3) were transported into cells against tumor cell lines such as NCI-H838 (lung adenocarcinoma), MES-SA (uterine carcinoma), HCT-116 (colon carcinoma), and NPC-TW01 (nasopharyngeal carcinoma) with the exception of breast cancer cells, based on Glut1-mediated transport, and then exhibited the cytotoxic activity [15]. Similarly, it is suggested that glucose-conjugated paclitaxel derivatives can be transported into breast cancer cells and exhibit the cytotoxic activity there, because GluT1 is overexpressed even in breast cancer cells. Furthermore, the amino acid transporter SLC6A14 $\left(\mathrm{ATB}^{0},{ }^{+}\right)$, whose substrates were neutral and cationic amino acids, was upregulated in colon cancer, cervical cancer, pancreatic cancer, and breast cancer. Interestingly, SLC6A14 was specifically expressed only in estrogen receptor (ER)-positive breast cancer, not in ER-negative breast cancer.

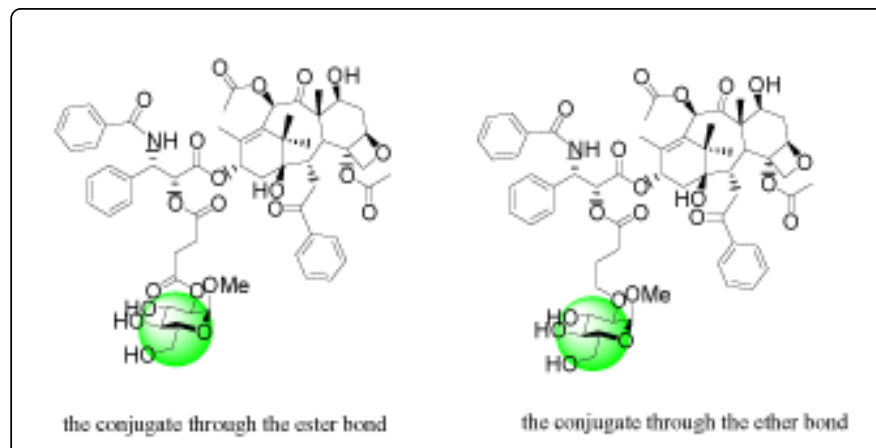

Figure 3: The structures of glucose-conjugated paclitaxel derivatives. Glucose mimics indicated by a green sphere might act as GluT1 recognition unit.

Butyrate and propionate are histone deacetylase (HDAC) inhibitors [16] and elicit anti-cancer effects. The conjugates composed of serine and HDAC inhibitors (butyrate and propionate) tethered by the ester bond (Figure 4) were transported into cells in in vitro assay using human retinal pigment epithelial cells and Xenopus laevis oocytes expressing human SLC6A14. Such conjugates are expected to elicit 
anti-cancer effects against ER-positive breast cancer, although data were not shown [17].

\section{Conclusions}

Cancer chemotherapy must resolve not only drug delivery into cells across the cell membrane but also acquired drug resistance through the overexpression of MDR1. The use of the transport system based on SLC transporters can overcome both problems. It is well-known that cancer cells overexpress some types of SLC transporters compared to normal cells or other types of cancers. Thus, compounds possessing transporter recognition unit of arbitrary SLC transporters penetrate the cell membrane, even though MDR1s are overexpressed (Figure 1). Accordingly, transporter-conscious drug design is an excellent tactic for effective cancer chemotherapy and good ADMET. In design of compounds transported by arbitrary SLC transporters, incorporated structural commonality of their substrates into designed compounds act as transporter recognition unit [3]. Compounds possessing peptide mimic as transporter recognition unit may enter into cancer cells overexpressing PEPT1. Compounds possessing glucose mimic as transporter recognition unit may enter into cancer cells overexpressing GluT1. Furthermore, compounds possessing anionic moiety as transporter recognition unit may enter into cancer cells overexpressing OATP1B1 or OATP1B3. In fact, such transporter-consciously designed compounds were delivered into cancer cells including breast cancer due to the transport systems and exhibited anti-cancer activity $[10,14,17]$.

Researchers, especially synthetic chemists and medicinal chemists, are expected to produce well-designed drugs based on transporterconscious design. Patients currently suffering from refractory cancers will be cured by chemotherapy using transporter-consciously designed drugs. In philosophical terms, the hard problem of consciousness means how qualia or phenomenal experiences such as colors and tastes are produced from the brain in material form. However, compounds in material form may move to the target organs within an organism using transport systems and metabolic systems as if they had consciousness and feared for MDR1, which are well designed by talented molecular designers.

\section{Acknowledgments}

This review is just my opinion based on or inferred from available published articles and public knowledge. Thus, the intellectual property rights are not infringed upon.

\section{References}

1. Nakanishi $\mathrm{T}$ (2007) Drug transporters as targets for cancer chemotherapy. Cancer Genomics Proteomics 4: 241-254.
2. Li Q, Shu Y (2014) Role of solute carriers in response to anticancer drugs. Mol Cell Ther 2: 15.

3. Tashima $\mathrm{T}$ (2015) Intriguing possibilities and beneficial aspects of transporter-conscious drug design. Bioorg Med Chem 23: 4119-4131.

4. International Transporter Consortium, Giacomini KM, Huang SM, Tweedie DJ, Benet LZ, et al. (2010) Membrane transporters in drug development. Nat Rev Drug Discov 9: 215-236.

5. Nakanishi T, Tamai I (2011) Solute carrier transporters as targets for drug delivery and pharmacological intervention for chemotherapy. J Pharm Sci 100: 3731-3750.

6. Kimura Y, Morita SY, Matsuo M, Ueda K (2007) Mechanism of multidrug recognition by MDR1/ABCB1. Cancer Sci 98: 1303-1310.

7. Alper J (2002) Drug delivery. Breaching the membrane. Science 296: 838-839.

8. Tashima T (2014) Multifunctionality of drug's pharmacophores within an organism. Drug Des. 3: 116.

9. Tsuji A (2002) Transporter-mediated Drug Interactions. Drug Metab Pharmacokinet 17: 253-274.

10. Fang L, Wang M, Gou S, Liu X, Zhang H, et al. (2014) Combination of amino acid/dipeptide with nitric oxide donating oleanolic acid derivatives as PepT1 targeting antitumor prodrugs. J Med Chem 57: 1116-1120.

11. Brown RS, Wahl RL (1993) Overexpression of Glut-1 glucose transporter in human breast cancer. An immunohistochemical study. Cancer 72: 2979-2985.

12. Hussein YR, Bandyopadhyay S, Semaan A, Ahmed Q, Albashiti B, et al. (2011) Glut-1 Expression Correlates with Basal-like Breast Cancer. Transl Oncol 4: 321-327.

13. Buxhofer-Ausch V, Secky L, Wlcek K, Svoboda M, Kounnis V, et al. (2013) Tumor-specific expression of organic anion-transporting polypeptides: transporters as novel targets for cancer therapy. Journal of Drug Delivery: ID 863539.

14. Kouodom M N, Ronconi L, Celegato M, Nardon C, Marchiò L, et al. (2012) Toward the selective delivery of chemotherapeutics into tumor cells by targeting peptide transporters: tailored gold-based anticancer peptidomimetics. J Med Chem 55: 2212-2226.

15. Lin YS, Tungpradit R, Sinchaikul S, An FM, Liu DZ, et al. (2008) Targeting the delivery of glycan-based paclitaxel prodrugs to cancer cells via glucose transporters. J Med Chem 51: 7428-7441.

16. Tashima T, Murata H, Kodama H (2014) Design and synthesis of novel and highly-active pan-histone deacetylase (pan-HDAC) inhibitors. Bioorg Med Chem 22: 3720-3731.

17. Bhutia YD, Babu E, Prasad PD, Vadivel Ganapathy V (2014) The amino acid transporter SLC6A14 in cancer and its potential use in chemotherapy. Asian J Pharm Sci 9: 293-303. 